\title{
Correction to: Systemic Inflammation as a Driver of Brain Injury: the Astrocyte as an Emerging Player
}

\author{
Bruna Bellaver ${ }^{1}$. João Paulo dos Santos ${ }^{1}$ - Douglas Teixeira Leffa ${ }^{2,3} \cdot$ Larissa Daniele Bobermin $^{1}$. \\ Ricardo Haack Amaral Roppa ${ }^{1}$ - Iraci Lucena da Silva Torres ${ }^{2,3}$ - Carlos-Alberto Gonçalves ${ }^{1}$ • \\ Diogo Onofre Souza ${ }^{1}$ - André Quincozes-Santos ${ }^{1}$
}

Published online: 26 May 2020

(C) Springer Science+Business Media, LLC, part of Springer Nature 2020

Correction to: Mol Neurobiol (2018) 55, 2685-2695

https://doi.org/10.1007/s12035-017-0526-2

The givenname "Paola" of the author Paola Haack Amaral Roppa (Roppa, P.H.A.) should be corrected to read Ricardo Haack Amaral Roppa (Roppa, R.H.A.) as presented above.

The online version of the original article can be found at https://doi.org/ 10.1007/s12035-017-0526-2

Bruna Bellaver

brunabellaver90@gmail.com

$\triangle$ André Quincozes-Santos andrequincozes@ufrgs.br

1 Departamento de Bioquímica, Programa de Pós-Graduação em, Ciências Biológicas: Bioquímica, Instituto de Ciências Básicas da Saúde, Universidade Federal do Rio Grande do Sul, Rua Ramiro, Barcelos, 2600-Anexo, Bairro Santa Cecília, Porto Alegre, RS 90035-003, Brazil

2 Programa de Pós-Graduação em Medicina: Ciências Médicas, Faculdade de Medicina, Universidade Federal do Rio Grande do Sul, Porto Alegre, RS, Brazil

3 Unidade de Experimentação Animal, Hospital de Clínicas de Porto, Alegre, Porto Alegre, RS, Brazil 